\title{
Ambiente informacional e desempenho competitivo na indústria de autopeças para veículos
}

\author{
Silvio Popadiuk \\ Marcos Antonio Franklin \\ Walter Miyabara \\ Roberto Gardesant \\ Universidade Presbiteriana Mackenzie
}

\begin{abstract}
Resumo
0 atual ambiente competitivo exige que as empresas tomem posturas diferentes em relação ao seu poder de obtenção de vantagem competitiva. A informação é o fator fundamental nesse cenário e, entre o seu input e o seu output, deveria ocorrer uma agregação de valor e ela, decorrente de um conjunto de processos relativos às tomadas de decisões. Neste contexto, com o presente trabalho, objetiva-se estudar o ambiente informacional de 50 empresas da indústria de autopeças para veículos e relacioná-lo com o desempenho competitivo, segundo a abordagem do balanced scorecard. Para tanto, foi aplicado um questionário estruturado fechado em empresas localizadas no Estado de São Paulo. Os resultados revelaram evidências de que as empresas classificadas como tendo um desempenho competitivo mais favorável tenderam a avaliar mais favoravelmente os atributos relativos ao ambiente informacional.
\end{abstract}

\section{Palavras-chave}

Ambiente informacional, desempenho competitivo, balanced scorecard, informação, autopeças.

\section{Informational environment and competitive performance in the automobile supply industry}

\begin{abstract}
The current competitive environment demands different postures from companies aiming at obtaining competitive advantage. In this scenery the information is the fundamental factor and, between its input and its output, should have an aggregation of value to it based on a set of processes related to decision making. From this point, this paper has been developed with the objective to study the informational environment and to verify connections with its competitive performance, according to Balanced Scorecard model. 50 automobile supply industries settled in São Paulo has been sampled and a structured questionnaire has been applied to them. The results has allowed to identify that, for companies where competitive performance were more favorable, the evaluation of the informational environment were quite different from the ones were competitive performance were considered less favorable.
\end{abstract}

\section{Key words}

Informational environment, competitive performance, balanced scorecard, information, automobile supply industry 


\section{INTRODUĈ̣̃O}

Num sistema que deseja gerar valor, é necessária a presença de um fator fundamental: a informação. Embora exista consenso sobre a importância estratégica da informação, a literatura disponível aborda principalmente o tratamento eletrônico dos dados e seu reflexo nos processos empresariais. $\mathrm{O}$ ambiente informacional das empresas que fazem parte da indústria de autopeças para veículos e seu relacionamento com os resultados operacionais das mesmas ainda é um tema pouco explorado, razão principal do desenvolvimento deste estudo, cujo objetivo é identificar as conexões entre atributos relativos ao ambiente informacional e o desempenho competitivo dessas empresas.

\section{FUNDAMENTAC̣̃̃O TEÓRICA}

\section{Informação sob o foco estratégico}

A sobrevivência e o sucesso de qualquer empresa dependem da vantagem competitiva (PORTER, 1989) que pode ser alcançada pela oferta de riquezas com custos baixos e benefícios diferenciados, pela adoção de novas tecnologias, novos métodos administrativos, pela utilização de recursos de forma racionalizada e integrada e pela inovação constante de produtos e serviços (WAHBA, 1993; HAMEL \& PRAHALAD, 1998; CHRISMAN et al., 1988; OLIVA et al., 1988; MAHONEY \& PANDIAN, 1992), criando uma base para a diferenciação e geração de

Figura 1: Dimensões do ambiente informacional. valor para o consumidor. A proposição de valor para o consumidor, como exemplo de uma atividade de relevância estratégica, consiste na combinação de três importantes fatores que irão diferenciar o produto ou serviço de uma empresa em relação à de seus concorrentes: a qualidade, o preço e os serviços oferecidos (TUCKER, 1999). Neste contexto, os sistemas de informação representam as ferramentas necessárias ao tratamento adequado da informação (ANDREU et al., 1996).

\section{Ambiente informacional}

$\mathrm{O}$ ambiente informacional está relacionado com o ambiente organizacional e com o ambiente externo da empresa (DAVENPORT, 2001). No ambiente organizacional, a situação dos negócios, os investimentos em tecnologia e a distribuição física repercutem e influenciam o ambiente informacional. No que se refere ao ambiente externo, aspectos como competitividade, existência ou não de soluções de TI disponíveis e tipos de informações normalmente comercializadas também influem diretamente no ambiente informacional interno que, conforme sugere Davenport (2001), envolve seis dimensões: a informação como uso estratégico; a política da informação; a cultura relacionada com o uso da informação, a equipe envolvida no desenvolvimento, treinamento e disseminação da informação; o processo de gerenciamento da informação e a arquitetura da informação. Para esse autor, este conjunto de dimensões seria o que ele define como um modelo ecológico, conforme se sintetiza na Figura 1.

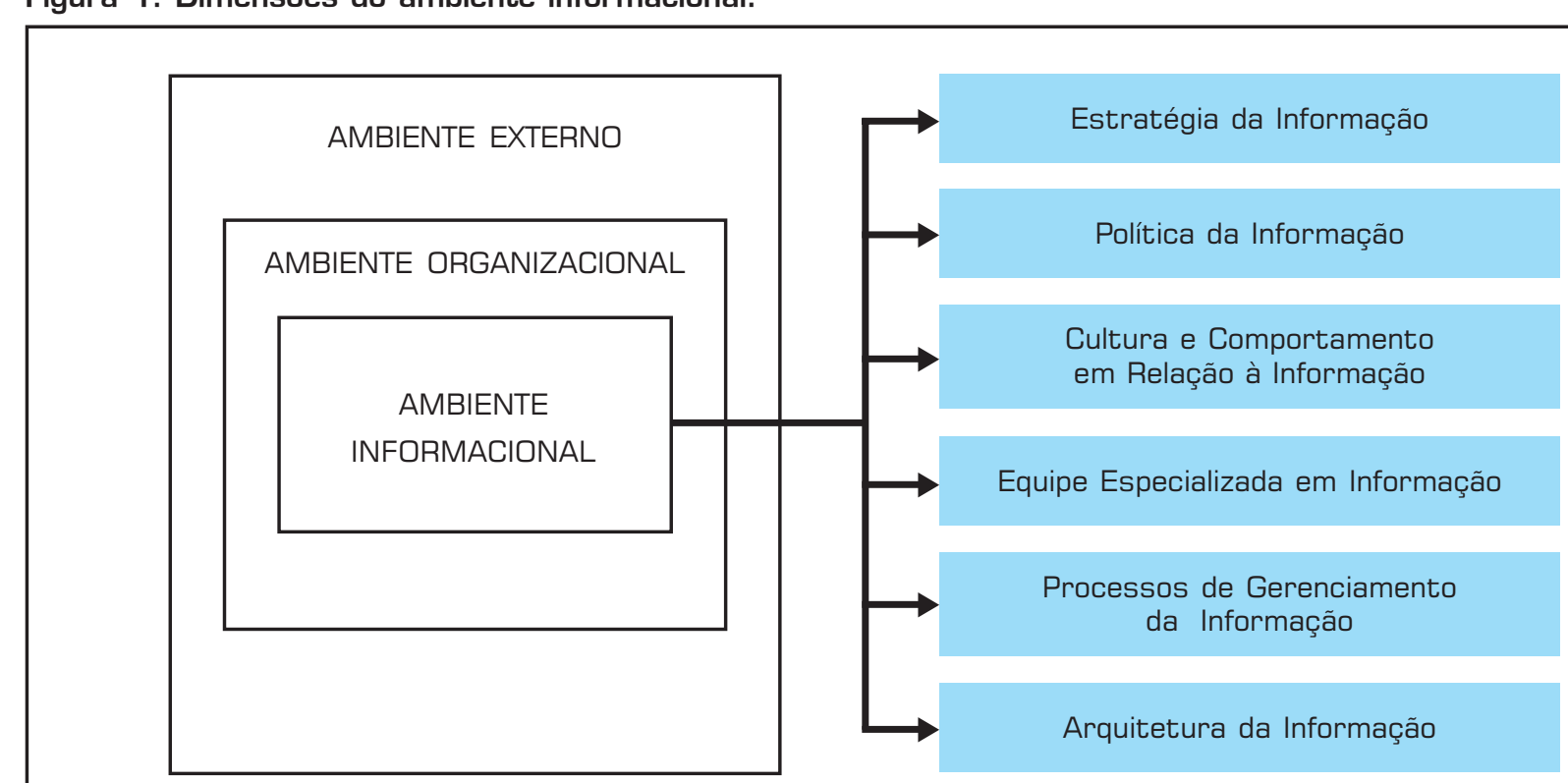

Fonte: Adaptado pelos autores a partir de Davenport (2001). 
Estratégia da informação - Segundo Prusak (1995), a informação como estratégia de negócio revela duas possibilidades reais que podem se tornar vitais em uma organização. A primeira é a identificação de alternativas inovadoras; a segunda é a identificação de situações que poderão interferir na sobrevivência da empresa, devido ao imprevisível movimento dos ambientes externo e interno, que devem estar alinhados com a estratégia empresarial. Em função das conjunturas apresentadas pelos ambientes, esse alinhamento deve ser dinâmico, assim como o mercado. Para Davenport (2001), a estratégia da informação deve deixar claras diversas questões referentes à informação, como, por exemplo, quais informações são importantes, permitindo um desempenho superior e vantagem competitiva sustentável (KETTINGER et al., 1994).

Política da informação - Davenport (2001) cita que, em praticamente todas as organizações, a informação é influenciada pelo poder, pela política e pela economia. Em modelos organizacionais de trabalho mais políticos, a informação tende a ser ambígua, sendo utilizada e sonegada estrategicamente, significando maior ou menor grau de centralização, conforme discutido por Souza \& Vasconcellos (2004), George \& King (1991), Menezes et al., (2003), Allen \& Boynton (1991), King (1983) e Von Simsom (1990).

\section{Num sistema que deseja gerar valor, é necessária a presença de um fator fundamental: a informação.}

Davenport (2001) comenta que existem diversos modelos possíveis de serem utilizados para governar a informação e cabe à organização determinar o que melhor se adapte. $\mathrm{O}$ autor apresenta quatro modelos - ou metáforas -, sendo que a grande diferença entre eles está na forma de controle central exercido pela empresa: a anarquia, o feudalismo, o federalismo e a monarquia. Além desses modelos, cita que existem critérios que não estão baseados em poder para definir o governo da informação: a) o modelo voltado para o mercado, onde a demanda pela informação é que define como ela deve ser dirigida, criando uma administração descentralizada e ausência de clareza em relação à prioridade e; b) o modelo chamado de utopia tecnocrática, cuja essência consiste na concepção de que a presença de tecnologias é suficiente para solucionar o problema de informações nas empresas.

Cultura e comportamento relacionados à informação - A cultura da informação é um dos elementos vitais para a empresa que procura mudança. Essa cultura pode ser definida como o conjunto de valores, atitudes e comportamentos que influenciam a forma como a pessoa avalia, apreende, recolhe, organiza, processa, comunica e utiliza a informação. Davenport (2001) define comportamento informacional, de forma sintética, como o modo como os indivíduos lidam com a informação. Salienta que deve incluir a busca, o uso, a alteração, a troca, o acúmulo e o ato de ignorar a informação.

Já a cultura informacional é definida como o comportamento que abrange grupos ou organizações. Representa valores e crenças, bem como o padrão de comportamento e atitude que expressam a orientação informacional de uma empresa. Pode ser fechada ou aberta, orientada por fatos ou baseada na intuição e/ou rumores, de enfoque interno ou externo, controlada ou autorizada e representar preferências por tipos de canais ou meios. Lederer e Mendelow (1988) abordam aspectos relacionados ao tema, focando-se na discussão sobre mecanismos de convencimento da alta gerência sobre a importância do potencial de sistemas de informações como ferramenta estratégica.

Segundo Davenport (2001), compartilhar informações é um ato voluntário de colocá-las à disposição de outros. O rodízio entre gerentes, reuniões freqüentes com gerentes de outros setores e decisões que ressaltem o consenso contribuem para esse compartilhamento, que precisa estar apoiado na cultura organizacional. Ele pode não ser exercido caso os profissionais enxerguem a informação como de valor para suas carreiras, caso algumas informações criem imagens negativas de pessoas ou setores e se o tempo gasto na transmissão da informação não for reconhecido e até recompensado.

A forma como a informação é divulgada e o envolvimento pessoal com ela implica na sobrecarga informacional. É necessária uma preocupação com o controle dos seus múltiplos significados. Significados alternativos geram resultados diferentes que podem não auxiliar no processo de tomada de decisão. Por outro lado, a existência de múltiplos significados representa que está havendo uma preocupação com a entidade em questão e representa um alerta, pois a definição formal pode não estar bem formulada ou definida; a padronização deveria permitir a aceitação de todos os envolvidos no processo.

Equipe de informação - Uma estrutura de apoio de qualquer ambiente informacional deve ser contemplada com pessoas que agreguem valor à informação. Esse grupo de pessoas é composto pelos programadores, analistas de sistemas, administradores de bancos de dados, gerentes de recursos de informações e outros profissionais que tenham uma compreensão abrangente da área de 
atuação e conhecimento da estrutura e função da empresa. Além disso, devem conhecer as diferentes fontes de informações da empresa, terem facilidade de acesso às tecnologias e fortes qualificações para relações interpessoais. Mais ainda, o foco totalmente voltado para o negócio ao invés de se voltarem aos objetivos funcionais da organização (DAVENPORT, 2001).

Gerenciamento da informação - Segundo Davenport (2001), o processo informacional deve ser analisado tanto pelo foco do gerador da informação, como também pela observação do usuário ou cliente da informação gerada. Para tanto, torna-se necessária a identificação do processo principal e posteriormente suas respectivas demandas com suas atividades básicas. Em outras palavras, os processos de gerenciamento da informação definem como a empresa obtém, distribui e utiliza a informação e, além disso, a conexão desses processos com o planejamento estratégico da organização (ROGERS et al., 1999).

Com esta proposta, Davenport (2001) menciona a necessidade de se identificarem: a) as necessidades de informação; b) a obtenção, a classificação e a formatação de acordo com o nível hierárquico do usuário na organização; c) a distribuição, onde são analisados os aspectos pertinentes à informação e sua ligação com gerentes e funcionários. Tais aspectos dizem respeito à estrutura política de acesso à informação, a tecnologia disponível e a planta arquitetônica que faz a ligação entre os processos, os comportamentos, a estrutura organizacional e o espaço físico, entre outros; d) uso da informação, que se refere aos aspectos ligados à identificação dos usuários que utilizam a informação, o seu emprego e o número de acessos.

Arquitetura da informação - A arquitetura da informação se constitui numa série de ferramentas que adaptam os recursos às necessidades da informação. Ela conecta os processos, os comportamentos, os métodos, a estrutura e o espaço físico, incluindo mapas, diretórios e padrões relacionados com o uso e armazenamento das informações. Na visão de Henderson e Sifonis (1988), o processo de planejamento de um ambiente informacional exige definição de fatores-chaves das necessidades de informação para os produtos e serviços e consistência no alinhamento entre as estratégias de negócio e as estratégias de TI.

Zachman (1987) definiu uma metodologia para organizar a informação nas organizações que teve muita aceitação, embora ainda existam muitas organizações que ignoram esses conceitos. Sugere que o projeto da arquitetura da informação seja concebido de forma aná- loga à construção de uma residência. Na área da informação organizacional, o projeto deve abordar as perspectivas dos dados, processos e tecnologia, evoluindo do desenho rudimentar, a partir de uma visão genérica da organização, passando pelos filtros das visões do usuário, do projetista, do implementador, da representação funcional detalhada e dos aspectos de tecnologia e de operacionalização. Isso significa que o projetista, chamado aqui de arquiteto da informação, deve auxiliar a organização no entendimento dos requisitos e desejos, partindo da visão mais generalista para a mais específica, abordando níveis de detalhamento adequados para cada fase do projeto e orientando a organização nas limitações, diretrizes e implicações de cada opção ou decisão.

\section{Desempenho competitivo - Balanced Scorecard}

Kaplan \& Norton (1997) afirmam que o Balanced Scorecard é um conjunto de indicadores que proporcionam uma visão rápida e abrangente da empresa, mesclando a perspectiva financeira com a perspectiva do cliente, perspectiva interna da empresa e perspectiva de inovação e aprendizado. Para esses autores, o Balanced Scorecard coloca no centro a estratégia e não os controles, além de fornecer aos gestores a informação necessária para decisões criadoras de valor, traduzindo visões e estratégias em objetivos.

A determinação de quais indicadores são mais adequados à companhia é realizada a partir da identificação das suas necessidades estratégicas. Para Kaplan (1999) não existem duas organizações iguais, e por isso cada uma segue o seu próprio caminho na criação de um conjunto apropriado de indicadores.

Para Paschoal (2003), uma só perspectiva não é suficiente para estabelecer os resultados integrais da corporação. Contudo, a perspectiva financeira fica melhor alicerçada sobre uma conexão com as demais perspectivas do Balanced Scorecard. O estabelecimento das demais perspectivas deve estar vinculado com os resultados financeiros, pois não pode ser escolhido um resultado financeiro que não seja derivado de todas as ações configuradas nas demais perspectivas. A perspectiva de clientes é a geradora de resultados que fará a sustentação do quadro financeiro.

A satisfação dos clientes é um índice que depende da realização de uma mensuração de opiniões que estão 
externas à empresa, que são geradas a partir de comportamentos internos das pessoas da empresa. Formatos de pesquisa e análise para mensurar o desempenho da empresa a partir do ponto de vista de seus clientes podem ser observados, por exemplo, em Hayes (1999), onde há uma indicação de que um modelo geral de desenvolvimento da mensuração da satisfação dos clientes pode ser realizado através de questionários, a partir da determinação das necessidades e exigências dos clientes.

Com o atual ajuste do foco da economia e das empresas para a necessidade de inovação e mudanças, amparadas pelo conhecimento organizacional, os processos, para serem melhorados, dependem da capacidade das pessoas que constituem a empresa. Assim, os processos internos passam a integrar outra perspectiva do Balanced Scorecard, que é a de aprendizado e crescimento. Para Kaplan e Norton (1997), a perspectiva de crescimento oferece a infra-estrutura que possibilita a consecução de objetivos ambiciosos nas outras três perspectivas. Os objetivos desta perspectiva, segundo os mesmos autores, são os vetores de resultados excelentes nas três primeiras perspectivas do scorecard. Os indicadores voltados para esta perspectiva, conforme Kaplan e Norton (1997), estão voltados à capacidade dos funcionários; capacidade dos sistemas de informação: motivação, empowerment e alinhamento. Para os funcionários, o grupo de medidas inclui sua satisfação, retenção e produtividade.

$\mathrm{O}$ aprendizado e crescimento das pessoas da organização são o alicerce da construção da evolução organizacional. A base do Balanced Scorecard é que toda a estratégia da empresa seja construída alicerçada nas pessoas, através de seus conhecimentos e aprendizados.

\section{PROCEDIMENTOS METODOLÓGICOS}

\section{Definições operacionais}

Ambiente informacional - $\mathrm{O}$ ambiente informacional foi operacionalizado nas seis dimensões, conforme a abordagem proposta por Davenport (2001), apresentadas em parágrafos anteriores. Dessa maneira, foram elaborados oito atributos para a estratégia da informação, 21 para a política da informação, 12 para a cultura e comportamento, 5 para a equipe, 6 para o gerenciamento e 15 atributos para a dimensão da arquitetura. Esses atributos estão enunciados nas Tabelas 2 a 7, utilizadas na análise dos dados.

Indicadores de desempenho competitivo - Para a operacionalização dos indicadores de desempenho competitivo foi utilizado o contexto do modelo do Balance Scorecard (KAPLAN \& NORTON, 1997) que sugerem a análise de quatro grandes dimensões, envolvendo os clientes, os processos, as finanças e o aprendizado orga- nizacional. Algumas adaptações desse modelo conceitual foram feitas com a finalidade de viabilizar a coleta dos dados. Entendia-se que determinados indicadores das empresas eram de caráter estratégico e, portanto, confidenciais, dificultando a sua obtenção. Dessa forma, nesta pesquisa, foram utilizados indicadores subjetivos, avaliados numa escala tipo Likert com cinco pontos.

\section{Planejamento da amostra e coleta dos dados}

O planejamento da amostra contemplou um conjunto de 50 empresas da indústria de auto-peças para veículos. Para a obtenção do cadastro de empresas foram utilizadas várias fontes: Sindipeças, Federação das Indústrias, sites especializados em indicadores econômicos, revistas com foco empresarial e os jornais Valor e Gazeta Mercantil. Esse conjunto de empresas ficou delimitado, geograficamente, nos municípios de São Paulo, ABCD, Osasco e Guarulhos, localizados no Estado de São Paulo.

$\mathrm{O}$ instrumento de coleta consistiu num questionário estruturado fechado, constituído de atributos associados ao ambiente informacional e indicadores de desempenho competitivo, conforme se apresenta adiante. O questionário foi pré-testado junto a uma amostra de cinco empresas, permitindo-se identificar eventuais problemas decorrentes de estrutura lógica das questões, conceitos utilizados e quantidade de questões. A coleta de dados foi realizada durante os meses de agosto e setembro de 2003.

\section{Ambiente de estudo - a indústria de autopeças para veículos}

A produção de veículos no Brasil cresceu de 1.073.861 veículos em 1992 para 1.792.660 veículos em 2002. As vendas internas no mesmo período cresceram de 764.016 para 1.487.657 veículos e as exportações cresceram de 341.900 para 414.790 veículos. Existiam em 2002, 489 empresas associadas ao Sindipeças, das quais $72,7 \%$ tinham capital registrado nacional, $30,9 \%$ capital estrangeiro e as restantes, capital misto. A maior participação estrangeira era a dos Estados Unidos, com 31,1\% do total do capital estrangeiro, seguidos de: Alemanha, com 24,88\%; França, com 6,3\%; Itália, com 5,9\%; Espanha, com 5,28\%; Japão, com 4,6\%; Reino Unido, com 2,8\%; e outros países com $19,8 \%$. A maior parte delas tem mais de 10 anos de fundação e está localizada no Estado de São Paulo (na Cidade de São Paulo, 21,5 \%, Interior de São Paulo, 21,0\%, ABCD, 15,2\%, Restante da Grande São Paulo, 15,8 \%), e 26,6\% nos outros Estados. O faturamento da indústria de autopeças para veículos, em 2002, foi de US\$ 10,920 bilhões, 2,4\% do PIB do Brasil. As vendas foram efetuadas para as montadoras $(57,0 \%)$, a reposição $(18,0 \%)$, as exportações $(19,0 \%)$, intersetoriais $(6,0 \%)$. A balança comercial da indústria foi negativa em 
US\$ 95,3 milhões em 2002, resultado de US\$ 3.881,6 milhões de exportações e US\$ 3.979,9 milhões de importações. Os investimentos em 2002 foram de US\$ 260,0 milhões. 168 mil trabalhadores estavam empregados nessa indústria e a ociosidade média em relação à capacidade total foi de $36 \%$ (SINDIPEÇAS, 2004).

\section{TRATAMENTO E ANÁLISE DOS DADOS}

\section{Descrição da amostra}

Pela análise da amostra identificou-se que 56\% tinham estrutura administrativa familiar; para $78 \%$ delas o capital social era predominantemente nacional; $76 \%$ adotavam o modelo organizacional vertical/pirâmide, $10 \%$ o modelo organizacional por produtos e $14 \%$ outros modelos organizacionais; $38 \%$ foram classificadas como líderes de mercado, $10 \%$ ocupavam a $2^{\mathrm{a}}$ posição, $20 \%$ outra posição; $22 \%$ não exportavam, $62 \%$ exportavam até $25 \%$ do faturamento e $16 \%$ exportavam mais de $25 \%$ do faturamento.

\section{Modelagem para identificar grau de desempenho}

Para analisar os dados, de tal forma que fosse possível o relacionamento entre os dois construtos especificados na pesquisa - o ambiente informacional $e$ o desempenho competitivo - foi necessário o uso de técnicas de análises estatísticas multivariadas, envolvendo a análise fatorial e cluster analysis, tanto para um como para o outro construto. Como o desempenho das empresas não foi avaliado segundo indicadores contábeis, devido às dificuldades de se obter essas informações das empresas, procurou-se utilizar um conjunto de indicadores baseados na avaliação subjetiva dos informantes sobre a saúde da empresa, no ano de 2002 comparativamente com o ano de 2001. Com base no conjunto de indicadores utilizados, dentro do conceito de balanced scorecard, envolvendo as dimensões associadas com características financeiras, gestão de processos, aprendizagem e clientes, foi possível classificar as empresas em duas tipologias de desempenho competitivo: uma que poderia ser considerada menos favorável, especificada na análise como baixo desempenho, e outra considerada mais favorável, especificada na análise como alto desempenho.

Como a escala de avaliação era do tipo Likert, com cinco pontos, uma avaliação mais próxima de 1 revelaria uma maior desfavorabilidade do indicador no ano de 2002 ao ser comparado como 2001. Por outro lado, uma avaliação mais próxima de 5 revelaria uma maior favorabilidade do indicador em 2002, comparativamente com 2001. Dessa maneira, mesmo que um informante de uma determinada empresa tivesse associado o menor escore - valor 1 - para todos os atributos de desempenho competitivo, não necessariamente isto significaria que a empresa posicionou-se num patamar inferior ao daquela cujo informante atribuiu valores no limite superior da escala - valor 5. Devido a juízos de valor, decorrentes das políticas e estratégias adotadas por cada organização, a avaliação subjetiva de desempenho é relativa a essas políticas e estratégias. No entanto, para efeito do objetivo deste trabalho essa subjetividade de avaliação não deve ter impactado a classificação em um dos dois segmentos.

\section{ambiente informacional está relacionado ambiente organizacional e com 0 externo da empresa.}

Para os indicadores agregados no mesmo fator, segundo a carga fatorial, foram calculados os respectivos Alfas de Cronbach, com o objetivo de verificar a possibilidade de utilização da escala somada. Os valores obtidos para essa estatística revelaram-se adequados, superiores a 0,7. Considerando-se as escalas somadas para cada fator relacionado com o desempenho competitivo, foi feita uma análise de cluster com a finalidade de separar as empresas em dois conjuntos: aquelas consideradas como possuindo um desempenho mais favorável e aquelas possuindo um desempenho menos favorável. As respectivas médias de cada indicador de desempenho competitivo, para os dois conjuntos de empresas, bem como os devidos alfas de Cronbach são consolidados na Tabela 1.

Pelos resultados apresentados na Tabela 1, atividades terceirizadas, custo-entrega-devolução e desperdício de matéria-prima foram os três únicos indicadores considerados não significantes a $10 \%$ ao se comparar as empresas classificadas em alto e baixo desempenho, indicando que eles não eram relevantes no conjunto de atributos utilizados na análise de cluster para a separação em dois grupos. Contudo, preferiu-se incorporá-los no modelo de classificação, visando evitar perda de informações. Ressalta-se que a significância para os demais itens está naturalmente consistente, pois a natureza do processo de classificação provoca este tipo de diferenciação, isto é, máxima variância entre grupos e mínima variância dentro dos grupos. 


\section{Avaliação da relação entre o ambiente informacional e o desempenho competitivo}

As tabelas a seguir referem-se aos escores médios dos atributos relativos ao ambiente informacional, de acordo com cada uma das seis dimensões consideradas, considerando-se empresas classificadas no grupo de baixo ou de alto desempenho competitivo. Para cada dimensão do ambiente informacional foi feita uma análise fatorial visando identificar fatores que facilitassem a interpretação das análises.

Estratégia da informação e desempenho competitivo - Pela Tabela 2 pode-se verificar que a análise fatorial para esta dimensão permitiu identificar três fatores: gestão de TI, alinhamento da informação e centralização da estratégia da informação. Dos oito atributos pertinentes a essa dimensão, o teste de hipótese revelou evidências de que, para quatro deles, as médias dos escores eram diferentes ao nível de 10\% de significância, ao se comparar os valores das empresas classificadas em alto e baixo desempenho competitivo.

Há uma clara tendência de os valores obtidos serem mais próximos do limite superior da escala para as empresas classificadas no grupo de alto desempenho competitivo. Importante destacar a consistência dos resultados ao se comparar com o atributo relativo à elaboração das estratégias de informação. Como os escores médios para os três atributos anteriores considerados significativamente diferentes são relativamente menores no grupo de empresas classificadas como baixo desempenho, isto revela uma certa centralização da informação nas gerências. Em outras palavras, isso pode significar que as empresas classificadas nesse grupo tendem a ter as suas informações mais centralizadas. Entretanto, isso não significa que essa seja uma situação desfavorável, pois essa característica pode ser relativa ao perfil da organização pesquisada.

Política da informação e desempenho competitivo Pela Tabela 3 identifica-se que os atributos da política da informação foram agregados em sete fatores. Para um desses fatores, o compartilhamento de informações, nota-se que o escore médio tendeu a ficar num ponto intermediário da escala, revelando que na indústria de autopeças para veículos a resistência para a troca de

Tabela 1: Alfas de Cronbach para os fatores relacionados com os indicadores de desempenho e respectivas médias - Escala somada

\begin{tabular}{|c|c|c|c|c|}
\hline & \multirow{2}{*}{$\begin{array}{l}\text { ALFA DE } \\
\text { CRONBACH }\end{array}$} & \multicolumn{3}{|c|}{ MÉDIAS PARA O GRUPO COM DESEMPENHO: } \\
\hline & & BAIXO & ALTO & SIGNIFIGÂNCIA \\
\hline \multicolumn{5}{|l|}{ Financeiros } \\
\hline Rentabilidade & 0,895 & 2,46 & 3,40 & 0,000 \\
\hline Liquidez - solvência & 1,000 & 3,16 & 4,03 & 0,002 \\
\hline \multicolumn{5}{|l|}{ Gestão de clientes } \\
\hline Conquista de mercado & 0,800 & 3,08 & 3,65 & 0,000 \\
\hline Satisfação de clientes & 0,726 & 3,07 & 3,58 & 0,001 \\
\hline Novos produtos - serviços & 1,000 & 3,11 & 3,81 & 0,002 \\
\hline Participação exportação & 1,000 & 2,89 & 3,32 & 0,022 \\
\hline \multicolumn{5}{|l|}{ Gestão de processos internos } \\
\hline Inovação e P/D & 0,707 & 2,92 & 3,58 & 0,000 \\
\hline Atividades terceirizadas & 0,653 & 2,95 & 3,03 & 0,704 \\
\hline Qualidade e logística & 0,744 & 3,26 & 4,03 & 0,000 \\
\hline Custo/entrega/devolução & 0,600 & 3,19 & 3,39 & 0,239 \\
\hline Desperdício matéria-prima & 1,000 & 2,37 & 2,52 & 0,572 \\
\hline Capacidade instalada & 1,000 & 3,16 & 3,61 & 0,025 \\
\hline \multicolumn{5}{|l|}{ Aprendizagem e crescimento } \\
\hline Capacitação funcional & 0,733 & 3,05 & 3,63 & 0,002 \\
\hline Valorização da equipe & 0,680 & 2,89 & 3,53 & 0,000 \\
\hline Satisfação da equipe & 1,000 & 2,84 & 3,48 & 0,000 \\
\hline Rotatividade de pessoal & 1,000 & 3,37 & 2,81 & 0,029 \\
\hline TOTAL & & $38,0 \%$ & $62,0 \%$ & $100,0 \%$ \\
\hline
\end{tabular}


informações não é um fato tão preponderante. Verificase também que, nas empresas classificadas como tendo alto desempenho, os conflitos relacionados à troca de informações tendem a ser menores, bem como o desencontro de informações, ao se comparar com as empresas do outro grupo. Naquelas empresas há uma tendência maior para que os gerentes controlem o ambiente informacional e existe uma padronização de termos em toda a empresa. Mais ainda, os relatórios têm formatos adequados às necessidades da área.

Cultura e comportamento em relação à informação e desempenho competitivo - A análise fatorial aplicada aos atributos dessa dimensão permitiu identificar quatro fatores, conforme se apresenta na Tabela 4. Foram identificados vários atributos cujos escores médios evidenciaram diferenças entre os dois conjuntos de empresas. Identifica-se que as empresas classificadas no grupo de alto desempenho tenderam a serem mais concordantes de que o compartilhamento de informações ocorre com mais intensidade. Nessas, há objetividade na disseminação das informações, existem eventos formais e informais para integrar os empregados, facilita-se a forma de apresentação dos dados e considera-se o comportamento ao se desenvolver ambientes informacionais. Esses resultados revelam que as empresas classificadas no grupo de alto desempenho tendem a ser mais focadas na cultura e comportamento relativo às informações.

Equipe de informação e desempenho competitivo Do conjunto de atributos relativos a essa dimensão, a análise fatorial permitiu identificar apenas um fator, interpretado como competência da equipe de informação. Verifica-se, na Tabela 5, que as empresas classificadas como tendo alto desempenho tenderam a atribuir escores médios mais altos aos cinco atributos relativos a essa dimensão. Essa constatação revela uma grande consistência com os resultados das tabelas anteriores, porque se identificam nesse grupo de empresas as competências das equipes com relação à informação.

Gerenciamento da informação e desempenho competitivo - Dois fatores foram identificados nessa dimensão, conforme Tabela 6. A orientação para o cliente interno é mais marcante dentre as empresas que foram classificadas como possuindo alto desempenho. Essas tenderam a concordar que procuram conhecer as necessidades dos clientes internos na geração de informações, que se preocupam em identificar se a informação disseminada teve utilidade e desenvolver relatórios com sistemas de busca. Entretanto, verifica-se que, quanto à prontidão das informações, há evidências de que as informações chegam com atraso para as tomadas de decisões.

Uma hipótese para explicar essa aparente contradição relaciona-se com o grau de agilidade de tomada de decisão pela empresa. Pode-se supor que à medida que a empresa é mais competitiva, mais ágil ela tenha que ser para tomar decisões e, assim, a gerência pode entender que o tempo entre a solicitação, a elaboração e o recebimento da informação esteja mais lento do que o mesmo processo dentro das empresas classificadas como tendo baixo desempenho. Outra hipótese para explicar esse fenômeno talvez esteja relacionada com a arquitetura da

Tabela 2: Estratégia da informação e desempenho competitivo.

\begin{tabular}{|c|c|c|c|c|}
\hline \multirow{2}{*}{$\begin{array}{l}\text { ATRIBUTOS AVALIADOS PARA } \\
\text { ESTRATÉGIA DA INFORMAÇẪO }\end{array}$} & \multicolumn{3}{|c|}{$\begin{array}{l}\text { MÉDIA DO ATRIBUTO } \\
\text { SEGUNDO DESEMPENHO }\end{array}$} & \multirow{2}{*}{$\begin{array}{l}\text { SIGNIFI. } \\
\text { CÂNCIA }\end{array}$} \\
\hline & $\begin{array}{l}\text { CARGA } \\
\text { FATORIAL }\end{array}$ & BAIXO & ALTO & \\
\hline \multicolumn{5}{|l|}{ Gestão da TI (pessoas, processos e tecnologia) } \\
\hline O forte nessa empresa é sua tecnologia. & 0,859 & 4,05 & 4,30 & 0,329 \\
\hline Desenvolvem-se talentos que avaliam e agregam valor às informações. & 0,768 & 4,00 & 4,71 & 0,069 \\
\hline A estratégia da informação envolve diálogo com a equipe. & 0,633 & 4,16 & 4,65 & 0,195 \\
\hline Os recursos de Tl são alocados de acordo com as necessidades. & 0,609 & 4,47 & 4,81 & 0,361 \\
\hline \multicolumn{5}{|l|}{ Alinhamento da informação (interno e externo) } \\
\hline O principal enfoque do negócio é o cliente. & 0,857 & 5,53 & 5,68 & 0,333 \\
\hline As diretrizes relativas à informação são definidas a todo momento. & 0,760 & 3,63 & 4,32 & 0,081 \\
\hline Existem pessoas que têm o papel de transmitir informações. & 0,677 & 4,11 & 4,81 & 0,053 \\
\hline \multicolumn{5}{|l|}{ Centralização da estratégia da informação } \\
\hline As estratégias da informação são elaboradas pelos gerentes. & 0,934 & 5,32 & 4,52 & 0,013 \\
\hline TOTAL & & 19 & 31 & 50 \\
\hline
\end{tabular}


informação que, nesse caso, associa-se com os recursos alocados a ela.

Arquitetura da informação e desempenho competitivo - Para essa dimensão foram identificados quatro fatores, apresentados na Tabela 7 .

Ao se verificar todos os atributos relativos a essa dimensão, identifica-se que todos os valores dos escores médios tendem a ser maiores para as empresas classificadas no grupo de alto desempenho competitivo, revelando que esse grupo de empresas está mais envolvido com as questões relativas a essa dimensão. Destaca-se que a alta administração tem o conhecimento do desenvolvimento dessa arquitetura e, além disso, nesse desenvolvimento a mudança comportamental é prioridade.

\section{CONCLUSÕES E LIMITAC̣ÕES}

Conclusões - Para efeito de verificação de eventuais associações entre os dois construtos elaborados neste trabalho, visando atender ao seu objetivo principal, a identificação dos reflexos que o desempenho competitivo gera no ambiente informacional das empresas, os

Tabela 3: Política da informação e desempenho competitivo

\begin{tabular}{|c|c|c|c|c|}
\hline \multirow{2}{*}{$\begin{array}{l}\text { ATRIBUTOS AVALIADOS PARA } \\
\text { POLÍTIGA DA INFORMAÇÃO }\end{array}$} & \multicolumn{3}{|c|}{$\begin{array}{l}\text { MÉDIA DO ATRIBUTO } \\
\text { SEGUNDO DESEMPENHO }\end{array}$} & \multirow{2}{*}{$\begin{array}{l}\text { SIGNIFI- } \\
\text { GÂNGIA }\end{array}$} \\
\hline & $\begin{array}{l}\text { CARGA } \\
\text { FATORIAL }\end{array}$ & BAIXO & ALTO & \\
\hline \multicolumn{5}{|l|}{ Barreiras à troca de informaçóes } \\
\hline Existem resistências para se trocar informações. & 0,760 & 3,79 & 3,32 & 0,258 \\
\hline Uma área controla a maioria das informações do negócio. & 0,746 & 4.05 & 3,94 & 0,789 \\
\hline Há resistências para a troca de informações entre as áreas. & 0,729 & 4.00 & 3,35 & 0,103 \\
\hline Existem conflitos relacionados com a troca de informações. & 0,600 & 3,74 & 3,03 & 0,078 \\
\hline \multicolumn{5}{|l|}{ Variedade de fontes de informação } \\
\hline Muitas pessoas possuem seu próprio sistema de informações. & 0,879 & 3,00 & 3,10 & 0,814 \\
\hline Existem várias fontes de informações sobre o mesmo objeto de avaliação. & 0,645 & 3,21 & 3,26 & 0,915 \\
\hline Existem conflitos relacionados com a troca de informações entre as pessoas. & 0,644 & 3,74 & 2,90 & 0,030 \\
\hline Identifica-se que as informações são desencontradas. & 0,487 & 3,53 & 2,61 & 0,033 \\
\hline \multicolumn{5}{|l|}{ Diretrizes de utilização das tecnologias de informação } \\
\hline Acredita-se que a tecnologia possa resolver os problemas de informações. & 0,835 & 3,89 & 3,94 & 0,925 \\
\hline A área de informática controla o acesso à informação. & 0,762 & 3,95 & 4,19 & 0,598 \\
\hline O tipo de atividade estimula a criação de sistemas de informações particulares. & 0,624 & 2,63 & 2,90 & 0,569 \\
\hline A maioria das informações é administrada por unidades locais. & 0,547 & 4,26 & 4,32 & 0,880 \\
\hline \multicolumn{5}{|l|}{ Compreensão do uso da informação } \\
\hline Os gerentes controlam o ambiente informacional. & 0,871 & 3,74 & 4,77 & 0,020 \\
\hline 0 acesso às informações é livre. & 0,701 & 4,21 & 4,58 & 0,370 \\
\hline Qualquer termo utilizado tem o mesmo significado em toda a empresa. & 0,532 & 3,74 & 5,10 & 0,000 \\
\hline \multicolumn{5}{|l|}{ Centralização de relatórios } \\
\hline Os relatórios têm formatos que atendem às necessidades da área. & 0,846 & 4,47 & 5,35 & 0,080 \\
\hline As unidades emitem relatórios de desempenho à unidade central. & 0,803 & 4,63 & 4,65 & 0,974 \\
\hline \multicolumn{5}{|l|}{ Estrutura e centralização do AI } \\
\hline A estrutura facilita a centralização do ambiente informacional. & 0,737 & 4,53 & 4,78 & 0,288 \\
\hline Existe pelo menos uma pessoa que centraliza o fluxo de informações. & 0,556 & 4,00 & 4,32 & 0,444 \\
\hline Identificam-se insatisfações relacionadas com o ambiente informacional. & 0,545 & 4,37 & 3,97 & 0,245 \\
\hline \multicolumn{5}{|l|}{ Fonte de poder } \\
\hline A informação traz poder ao informante. & 0,895 & 4,26 & 3,71 & 0,199 \\
\hline TOTAL & & 19 & 31 & 50 \\
\hline
\end{tabular}


escores médios de cada atributo, dentro de cada dimensão, foram comparados controlando-se o grupo de desempenho competitivo, na indústria de autopeças para veículos. As principais conclusões, apresentadas a seguir, são segmentadas de acordo com as seis dimensões relacionadas com o ambiente informacional, sintetizadas na Tabela 8 .

Por essa tabela verifica-se que, de todas as comparações dos atributos considerando-se os dois conjuntos de empresas, pelo menos $52,2 \%$ puderam ser considerados estatisticamente diferentes. O grande destaque é a dimensão relativa à equipe de informação onde todas as comparações foram consideradas diferentes estatisticamente.

Na dimensão relativa à estratégia da informação, as empresas classificadas no grupo de alto desempenho competitivo tenderam a avaliar de forma mais concor-

Tabela 4: Cultura e comportamento em relação à informação e desempenho competitivo.

\begin{tabular}{|c|c|c|c|c|}
\hline \multirow{2}{*}{$\begin{array}{l}\text { ATRIBUTOS AVALIADOS PARA CULTURA E } \\
\text { COMPORTAMENTO EM RELAÇÃO À INFORMAÇÃO }\end{array}$} & \multicolumn{3}{|c|}{$\begin{array}{l}\text { MÉDIA DO ATRIBUTO } \\
\text { SEGUNDO DESEMPENHO }\end{array}$} & \multirow{2}{*}{$\begin{array}{l}\text { SIGNIFI- } \\
\text { GÂNGIA }\end{array}$} \\
\hline & $\begin{array}{l}\text { CARGA } \\
\text { FATORIAL }\end{array}$ & BAIXO & ALTO & \\
\hline \multicolumn{5}{|l|}{ Compartilhamento de informações } \\
\hline A disseminação de informações pelas pessoas é feita com objetividade. & 0,821 & 4,21 & 4,81 & 0,078 \\
\hline Existem eventos formais de integração dos empregados. & 0,802 & 4,32 & 5,32 & 0,005 \\
\hline Procura-se apresentar as informações de forma simples e de fácil leitura. & 0,708 & 4,47 & 5,42 & 0,003 \\
\hline Ao desenvolver os ambientes informacionais, o comportamento é considerado. & 0,640 & 3,74 & 4,77 & 0,005 \\
\hline Existem eventos informais de integração dos empregados. & 0,597 & 3,89 & 4,90 & 0,010 \\
\hline Há preocupação em descobrir como as pessoas lidam com a informação. & 0,532 & 3,79 & 4,39 & 0,150 \\
\hline \multicolumn{5}{|l|}{ Comunicação com canal direto } \\
\hline Prefere-se comunicação de informações com a presença do informante. & 0,827 & 4,42 & 4,13 & 0,418 \\
\hline Acredita-se que os funcionários têm clareza sobre os objetivos da organização. & 0,713 & 3,63 & 4,90 & 0,001 \\
\hline Existe um programa para identificar talentos para atuar com informações. & 0,600 & 3,05 & 3,94 & 0,064 \\
\hline \multicolumn{5}{|l|}{ (Socialização) Troca da informação } \\
\hline Existem grupos de discussões sobre o ambiente informacional. & 0,800 & 3,37 & 4,06 & 0,128 \\
\hline Existem rodízios de gerentes e outros executivos. & 0,671 & 1,84 & 2,42 & 0,168 \\
\hline \multicolumn{5}{|l|}{ Padronização da comunicação da informação } \\
\hline Preferem-se comunicações de informações por sistemas impessoais. & 0,929 & 3,32 & 4,16 & 0,062 \\
\hline TOTAL & & 19 & 31 & 50 \\
\hline
\end{tabular}

Tabela 5: Equipe de informação e desempenho competitivo.

\begin{tabular}{|l|c|c|c|c|}
\multicolumn{2}{c|}{$\begin{array}{c}\text { ATRIBUtos AVALIADOS PARA } \\
\text { EQUIPE DE INFORMAÇÃO }\end{array}$} & \multicolumn{2}{c|}{$\begin{array}{c}\text { MÉDIA DO ATRIBUTO } \\
\text { SEGUNDO DESEMPENHO }\end{array}$} & $\begin{array}{c}\text { SIGNIFI- } \\
\text { GÂNCIA }\end{array}$ \\
\cline { 2 - 4 } & $\begin{array}{c}\text { GARGA } \\
\text { FATORIAL }\end{array}$ & BAIXO & ALTO & \\
\hline Competência da equipe de informação & & & & \\
\hline A equipe de informação tem compreensão da sua área de atuação. & 0,966 & 4,21 & 5,52 & 0,000 \\
\hline A equipe de informação conhece a estrutura da empresa. & 0,953 & 4,37 & 5,45 & 0,001 \\
\hline A equipe tem facilidade de acesso às tecnologias de informações. & 0,934 & 4,47 & 5,26 & 0,010 \\
\hline O perfil da equipe de informação voltado para o atendimento do usuário. & 0,811 & 4,16 & 5,16 & 0,003 \\
\hline A equipe de informação conhece as fontes de informações da empresa. & 0,853 & 4,16 & 5,29 & 0,001 \\
\hline TOTAL & & 19 & 31 & 50 \\
\hline
\end{tabular}


Tabela 7: Política da informação e desempenho competitivo.

\begin{tabular}{|c|c|c|c|c|}
\hline \multirow{2}{*}{$\begin{array}{l}\text { ATRIBUTOS AVALIADOS PARA } \\
\text { ARQUITETURA DA INFORMAÇÃO }\end{array}$} & \multicolumn{3}{|c|}{$\begin{array}{l}\text { MÉDIA DO ATRIBUTO } \\
\text { SEGUNDO DESEMPENHO }\end{array}$} & \multirow{2}{*}{$\begin{array}{l}\text { SIGNIFI- } \\
\text { GÂNGIA }\end{array}$} \\
\hline & $\begin{array}{l}\text { GARGA } \\
\text { FATORIAL }\end{array}$ & BAIXO & ALTO & \\
\hline \multicolumn{5}{|l|}{ Estrutura lógica da arquitetura do AI } \\
\hline $\begin{array}{l}\text { No desenvolvimento da arquitetura informacional a mudança } \\
\text { comportamental é prioridade. }\end{array}$ & 0,850 & 4,16 & 4,52 & 0,270 \\
\hline Existe a idéia de que o ambiente informacional deve ter um mapa lógico. & 0,838 & 3,74 & 4,29 & 0,167 \\
\hline O conteúdo lógico informacional é compreensível para a maioria dos empregados. & 0,694 & 3,68 & 4,42 & 0,053 \\
\hline $\begin{array}{l}\text { Utiliza-se uma abordagem planejada para identificar as informações não } \\
\text { eletrônicas. }\end{array}$ & 0,58 & 3,68 & 4,42 & 0,053 \\
\hline O mapa lógico possui documentação sobre conceitos e usos do sistema. & 0,642 & 3,37 & 4,48 & 0,012 \\
\hline Incentivos são incorporados para o sucesso do ambiente informacional. & 0,575 & 3,37 & 4,16 & 0,043 \\
\hline \multicolumn{5}{|l|}{ Integração e flexibilidade da arquitetura do AI } \\
\hline Nesta empresa preocupa-se mais com investimentos tecnológicos. & 0,824 & 3,42 & 4,42 & 0,009 \\
\hline As bases de dados da empresa permitem integração dos dados. & 0,588 & 4,37 & 5,26 & 0,002 \\
\hline O Al é desenvolvido de tal forma que seja flexível e mutável no tempo. & 0,523 & 3,84 & 4,65 & 0,014 \\
\hline \multicolumn{5}{|l|}{ Política de desenvolvimento da arquitetura do AI } \\
\hline A alta administração tem conhecimento do desenvolvimento do Al. & 0,734 & 5,00 & 5,52 & 0,011 \\
\hline É possível elaborar um mapa lógico do ambiente informacional. & 0,630 & 4,11 & 4,84 & 0,019 \\
\hline O desenvolvimento do ambiente informacional parte de baixo para cima. & $-0,575$ & 2,74 & 2,90 & 0,732 \\
\hline \multicolumn{5}{|l|}{ Homogenidade da arquitetura do Al } \\
\hline As informações ficam armazenadas em diversos meios e formatos. & 0,781 & 3,37 & 3,90 & 0,208 \\
\hline O desenvolvimento do ambiente informacional parte de cima para baixo. & 0,742 & 4,58 & 4,42 & 0,657 \\
\hline As informações encontram-se muito dispersas pela empresa. & 0,584 & 3,53 & 3,00 & 0,180 \\
\hline TOTAL & & 19 & 31 & 50 \\
\hline
\end{tabular}

Tabela 6: Gerenciamento da informação e desempenho competitivo.

\begin{tabular}{|c|c|c|c|c|}
\hline \multirow{2}{*}{$\begin{array}{l}\text { ATRIBUTOS AVALIADOS PARA } \\
\text { GERENGIAMENTO DA INFORMAÇÃO }\end{array}$} & \multicolumn{3}{|c|}{$\begin{array}{l}\text { MÉDIA DO ATRIBUTO } \\
\text { SEGUNDO DESEMPENHO }\end{array}$} & \multirow{2}{*}{$\begin{array}{l}\text { SIGNIFI- } \\
\text { GÂNGIA }\end{array}$} \\
\hline & $\begin{array}{l}\text { CARGA } \\
\text { FATORIAL }\end{array}$ & BAIXO & ALTO & \\
\hline \multicolumn{5}{|l|}{ Orientação ao cliente interno } \\
\hline $\begin{array}{l}\text { Procura-se conhecer necessidades dos clientes internos na geração de } \\
\text { informações. }\end{array}$ & 0,876 & 3,89 & 5,06 & 0,001 \\
\hline Há preocupação em identificar se a informação disseminada teve utilidade. & 0,868 & 3,84 & 4,90 & 0,007 \\
\hline Os relatórios existentes são classificados com sistema de busca. & 0,851 & 3,68 & 4,39 & 0,084 \\
\hline \multicolumn{5}{|l|}{ Prontidáo da informação } \\
\hline A maioria das informações informais chega tarde demais para a sua utilização. & 0,848 & 3,8 & 4,5 & 0,118 \\
\hline $\begin{array}{l}\text { A maioria das informações formais é de pouca utilidade para a tomada de } \\
\text { decisão. }\end{array}$ & 0,814 & 4,0 & 4,9 & 0,434 \\
\hline A maioria das informações formais chega tarde demais para a sua utilização. & 0,733 & 4,1 & 5,0 & 0,019 \\
\hline TOTAL & & 19 & 31 & 50 \\
\hline
\end{tabular}


dante os atributos relativos a essa dimensão, comparativamente aos valores das empresas classificadas no grupo de baixo desempenho, revelando um foco maior na estratégia da informação. Os resultados permitiram identificar que, para os dois grupos de empresas, o principal enfoque do negócio é o cliente. Entretanto, os atributos relativos ao desenvolvimento de talentos que avaliam e agregam valor às informações, a alocação de recursos de TI, a definição de diretrizes relativas à informação e a presença de pessoas que tenham o papel de transmitir informações pela organização foram avaliados mais favoravelmente dentre o conjunto de empresas classificadas no grupo de alto desempenho competitivo. Por outro lado, no grupo da empresas classificadas no grupo de baixo desempenho competitivo, os resultados revelaram uma certa tendência à centralização das informações, constatação reforçada pelo fato de que a gerência tende a ter um papel mais forte na elaboração das estratégias de informação das empresas, podendo significar um maior grau de centralização das informações, mas não necessariamente significando que essa situação seja desfavorável. Ela pode simplesmente ser consequiência do perfil da organização pesquisada.

$\mathrm{Na}$ análise dos 21 atributos relativos à política da informação, agrupados em sete fatores, ao se aplicar a análise fatorial, pode-se identificar que, nas empresas classificadas no grupo de alto desempenho competitivo, há um grau maior de descentralização das informações. Embora pela Tabela 8 possa-se verificar que nessa dimensão ocorreu o menor percentual de diferenças significantes $(28,6 \%)$, ao se comparar os valores médios dos dois grupos de empresas, os resultados apresentados em tabelas anteriores evidenciam que, nas empresas cujo desempenho competitivo foi classificado como baixo, é mais marcante a existência de conflitos relacionados à troca de informações, o desencontro de informações, uma certa falta de padrão de termos associados à informação e, além disso, há uma tendência para se avaliar mais categoricamente que a informação é uma fonte de poder para o informante. Outra constatação é que, para os dois grupos de empresas, não ocorre uma tendência à existência de sistemas de informações particulares, características da metáfora classificada como anarquia, discutida anteriormente.

\section{A determinação de quais indicadores são mais adequados à companhia ealizada a partir da identificação} suas necessidades estratégicas.

Para o conjunto de 12 atributos associados à dimensão da cultura e ao comportamento em relação à informação, consolidados em quatro fatores, após a aplicação da análise fatorial, foram identificadas diferenças significantes ao nível de $10 \%$, ao se comparar os valores médios dos atributos associados aos dois grupos de empresas. Verificou-se que, nas empresas classificadas no grupo de alto desempenho competitivo, a disseminação de informações é feita com mais objetividade, os eventos formais e informais visando a interação e integração dos empregados são mais constantes, o desenvolvimento do ambiente informacional considera os comportamentos e as informações são apresentadas de forma simples e de fácil leitura. Além disso, nesse grupo, os funcionários possuem mais clareza sobre os objetivos da organização, enfatizam-se mais os mecanismos para a identificação de talentos para lidar com informações e ocorre uma maior preferência para sistemas de comuni-

Tabela 8: Síntese dos resultados das significâncias encontradas.

\begin{tabular}{|l|c|c|c|}
\hline \multicolumn{1}{|c|}{$\begin{array}{c}\text { DIMENSÕES DO AMBIENTE } \\
\text { INFORMACIONAL }\end{array}$} & $\begin{array}{c}\text { (1) - ATRIBUTOS } \\
\text { COMPARADOS }\end{array}$ & $\begin{array}{c}\text { (2) - DIFERENÇAS } \\
\text { SIGNIFICANTES }\end{array}$ & $\begin{array}{c}\text { PERCENTUAL } \\
\text { (2)/(1) }\end{array}$ \\
\hline Estratégia da informação & 8 & 3 & 37,5 \\
\hline Política da informação & 21 & 6 & 28,6 \\
\hline Cultura e comportamento em relação à informação & 12 & 8 & 66,7 \\
\hline Equipe de informação & 5 & 5 & 100,0 \\
\hline Gerenciamento da informação & 6 & 4 & 66,7 \\
\hline Arquitetura da informação & 15 & 9 & 60,0 \\
\hline TOTAL & 67 & 35 & 52,2 \\
\hline
\end{tabular}


cação impessoais. Esses resultados revelam que as empresas classificadas nesse grupo tendem a ser mais focadas nos aspectos relativos à dimensão da cultura $\mathrm{e}$ informação, sugerindo a presença de iniciativas de gestão do conhecimento.

$\mathrm{Na}$ dimensão relativa à equipe de informação, para todos os cinco atributos, os valores médios foram considerados significativamente diferentes ao nível de $10 \%$, ao se comparar os dois grupos de empresas. Nas empresas classificadas no grupo de alto desempenho competitivo a equipe envolvida com assuntos relativos à informação tem uma melhor compreensão de sua área de atuação, conhece melhor a estrutura da empresa, tem mais facilidade de acesso às tecnologias, é mais voltada ao atendimento do usuário e revela melhor conhecimento sobre as fontes de informações da empresa.

Considerando-se a dimensão gerenciamento da informação, pôde-se verificar que, dentre as empresas classificadas no grupo de alto desempenho competitivo ocorre maior orientação a se gerenciar a informação com o foco no cliente interno. Assim, nesse grupo, há maior ênfase em conhecer as necessidades dos clientes internos, na geração de informações, na identificação da utilidade da informação disseminada e na caracterização mais adequada dos relatórios existentes. Por outro lado, quando a informação é disseminada, existe um alto grau de preocupação quanto a sua utilidade e aplicabilidade, principalmente na tomada de decisão quanto a sua prontidão. O tempo entre a sua solicitação, elaboração e seu recebimento é considerado mais longo dentre as empresas classificadas no grupo de alto desempenho. Embora seja uma aparente contradição com o exposto no início deste parágrafo, há uma possível explicação para este fato. Pode-se supor que as empresas desse grupo sejam mais ágeis do que as do outro grupo e, portanto, o tempo de resposta de suas demandas seja mais curto. Por essa razão, a avaliação dos atributos associados à prontidão das informações, um dos fatores dessa dimensão, foi mais desfavorável.

Na mesma direção das demais, na dimensão arquitetura da informação, para as empresas classificadas no grupo de alto desempenho os informantes tenderam a associar valores médios maiores do que os informantes das empresa do outro grupo e, para pelo menos nove dos quinze atributos, as diferenças foram consideradas estatisticamente significantes. Desse modo, para esse grupo de empresas, avaliou-se mais favoravelmente que o conteúdo lógico informacional é compreendido pela maioria dos empregados, há uma abordagem planejada para se identificar informações não eletrônicas, existe maior tendência à documentação de conceitos e usos do sistema, incentivos são utilizados visando o sucesso do ambiente informacional. Em adição, ocorre uma maior preocupação com investimentos em tecnologias de informações, com a integração de dados, com a flexibilidade dos sistemas existentes, e a alta administração tem o conhecimento do desenvolvimento do ambiente informacional.

Dessa forma, pode-se finalizar este estudo afirmandose que as empresas da indústria de autopeças para veículos cujo desempenho competitivo é classificado como alto revelaram fortes evidências de que o seu ambiente informacional é mais desenvolvido do que o das empresas classificadas no grupo de baixo desempenho, confirmando presença de associação entre os dois construtos desenvolvidos nesta pesquisa. Mais ainda, revela que para as empresas classificadas no grupo de alto desempenho competitivo, além de haver uma melhor gestão do ambiente informacional, caracterizam-se práticas que podem indicar a presença de um processo de gestão do conhecimento.

Limitações - Dentre o conjunto de limitações que podem reduzir o escopo das conclusões deste trabalho podem ser citadas aquelas relativas ao tamanho da amostra e a sua seleção, feita não aleatoriamente. Este fato, por si só, já não permitiria a aplicação dos testes estatísticos. Mesmo assim, preferiu-se aplicá-los, muito mais como um fator de redução da subjetividade de comparação do que a presença de significância estatística. Outro fator delimitador está relacionado com a operacionalização dos atributos relacionados com o ambiente informacional. Mesmo tendo sido tomados os devidos cuidados para a sua elaboração, com base na fundamentação teórica, ainda assim, pode ter havido algumas inconsistências relacionadas com a sua validade interna. Finalmente, uma outra preocupação que deve ter um pesquisador que utilizar este trabalho para estudos semelhantes é com relação aos indicadores de desempenho competitivo. Numa situação ótima eles deveriam ser avaliados a partir de dados contábeis, revelando com mais precisão a organização, segundo as dimensões do Balanced Scorecard.

Estudos futuros - Estudos futuros poderiam ser relacionados com a aplicação da pesquisa em atividades diferentes, controlando-se características das empresas e sua localização geográfica, utilizando-se amostras maiores e probabilísticas. 


\section{- Referências Bibliográficas}

ALLEN, B. R. ; BOYNTON, A. C. Information architecture: in search of efficient flexibility. MIS Quarterly, v.15, n.4, p. 435-445, Dec. 1991

ANDREU, Rafael; RICART, Joan E.; VALOR, Josep. Estratégia y sistemas de información. Madrid: McGraw-Hill, 1996.

CHRISMAN, James J.; HOFER, Charles W. ; BOULTON, William R. Toward a systems for classifying business strategies. Academy of Management Review. V. 134, n.3, p. 413028, 1988.

DAVENPORT, Thomas H. Ecologia da informação. São Paulo: Futura, 2001.

GEORGE, Joey; KING, John. Examining the computing and centralization debate. Communications of the ACM, v. 34 , n. 7, p. 62-72, July, 1991

HAMEL, Gary; PRAHALAD, C.K. Competindo pelo futuro. 15 ed. Rio de Janeiro: Campus, 1998.

HAYES, Bob E. Como medir la satisfacción del cliente: desarrollo y utilización de cuestionarios. 2 ed. Barcelona: Gestión 2000, 1999.

HENDERSON, John C.; SIFONIS, John $\mathrm{G}$. The value of strategic IS planning: understanding consistency, validity, and IS markets. MIS Quarterly, v. 12, n. 2, p. 187-200, Jun., 1988.
KAPLAN, Robert S. \& NORTON, David P. A estratégia em ação: balanced scorecard. Rio de Janeiro: Campus, 1997.

KAPLAN, Robert S. O balanced scorecard: gerenciando a performance futura. Belo Horizonte: Mindquest, 1999.

KETTINGER, William J.; GROVER, Varun; GUHA, Subashish; SEGARS, Albert H. Strategic information systems revisited: a study in sustainability and performance. MIS Quarterly, v. 18, n. 1, p. 31-58, March, 1994.

KING, J. L. Centralized versus decentralized computing: organizational considerations and management options. Computing Surveys, v. 15, n. 4, p. 320-49, Dec. 1983.

LEDERER, Albert L.; MENDELOW, Aubrey L. Convincing top management of the strategic potential of information systems. MIS Quarterly, v. 12, n. 4, p. 525-534, Dec. 1988.

MAHONEY, Joseph T.; PANDIAN, Rajendran J. The resource-based view within the conversation of strategic management. Strategic Management Journal, v. 13, n. 5, June, 1992.

MENEZES, Ricardo Bezerra; TEIXEIRA JUNIOR, Francisco; PONTE, Vera Maria Rodrigues. Centralização versus descentralização de tecnologia de informação: uma análise sob a ótica da eficácia organizacional. XXVII Encontro da Associação Nacional dos Programas de Pós Graduação - Anais ENANPAD, 2003.

OLIVA, Terence A.; DAY, Diana L.; MACMILLAN, Ian C. A generic model of competitive dynamics. Academy of Management Review. V. 13, n. 3, p. 374-89, 1988

PASCHOAL, Paulo Zanotto de. O uso de sistemas de gestão estratégica baseada em indicadores de desempenho pelas maiores empresas brasileiras: balanced scorecard $x$ sistemas de medição de desempenho da Fundação para o Prêmio Nacional da Qualidade - FPNQ. Dissertação (Mestrado em Controladoria e Contabilidade Estratégica) Centro Universitário Álvares Penteado: São Paulo, 2003.

PORTER, Michael E. Vantagem competitiva: criando e sustentando um desempenho superior. Rio de Janeiro: Campus, 1989.

PRUSAK, Larry. Gerenciamento da informação. São Paulo: Makron Books, 1995.

ROGERS, Patrick R.; MILLER, Alex; JUDGE, William Q. Using informationprocessing theory to understand planning/performance relationships in the context of strategy. Strategic Management Journal, v. 20, n. 6, p. 567-577, Jun., 1999.
SINDIPEÇAS. Desenvolvido pelo Sindicato Nacional da Industria de Componentes para Veículos Automotores. Apresenta informações sobre a indústria de componentes para veículos automotores. Disponível em: $<$ http: /www.sindipecas.org.br $>$. Acesso em 14 mar. 2004

SOUZA, César Alexandre de VASCONCELLOS, Eduardo Pinheiro Gondim. Tecnologia da informação e centralização organizacional: um estudo de caso de implementação de sistemas ERP. XXVIII Encontro da Associação Nacional dos Programas de Pós Graduação Anais ENANPAD, 2004

TUCKER, Robert B. Agregando valor ao seu negócio. São Paulo: Makron Books, 1999.

VON SIMSON, E. M. The centrally decentralized IS organization. Harvard Business Review, v. 68, n. 4, p. 158-62, July/Aug. 1990.

WAHBA, Claude. Rentabilidade: saindo da teoria e participando para a prática. São Paulo:Hamburg, 1993.

ZACHMAN, John A. A framework for information systems architecture. IBM Systems Journal, 26, 3, 1987.

\section{- Agradecimentos}

Agradecemos o apoio recebido do Fundo Mackenzie de Pesquisas para a realização deste estudo.

\section{- Sobre os autores}

\section{Silvio Popadiuk}

\section{Professor}

Universidade Presbiteriana Mackenzie

Endereço: Rua da Consolação, 896, 7o andar, Sala 76 - São Paulo - SP - 01302-907

Telefone: (11) 3236-8597 - E-mail: spopadiuk@mackenzie.com.br

\section{Marcos Antonio Franklin}

Professor

Universidade Presbiteriana Mackenzie

Endereço: Rua da Consolação, 896, 7o andar, Sala 76 - São Paulo - SP - 01302-907

Telefone: (11) 3236-8597 - E-mail: marcosfranklin@ mackenzie.com.br

\section{Walter Miyabara}

Professor

Universidade Presbiteriana Mackenzie

Endereço: Rua da Consolação, 896, 7ํandar, Sala 76 - São Paulo - SP - 01302-907

Telefone: (11) 3236-8597 - E-mail: waltermiyabara@terra.com.br

\section{Roberto Gardesani}

Professor

Universidade Presbiteriana Mackenzie

Endereço: Rua da Consolação, 896, 7o andar, Sala 76 - São Paulo - SP - 01302-907

Telefone: (11) 3236-8597 - E-mail: rgardesani@ mackenzie.com.br 